\title{
Profilaxia antimicrobiana em cirurgia vascular periférica: cefalosporina ainda é o padrão-ouro?
}

\author{
Antimicrobial prophylaxis in peripheral vascular surgery: is \\ cephalosporin still the gold standard?
}

\author{
Eduardo Lichtenfels ${ }^{1}$, Márcio L. Lucas², Ronaldo Webster ${ }^{3}$, Pedro A. d'Azevedo ${ }^{4}$
}

\section{Resumo}

Nas cirurgias vasculares periféricas, as cefalosporinas têm seu uso consagrado como agente antimicrobiano profilático de escolha. Recentemente, observamos uma mudança nos padrões de colonização, prevalência de patógenos e suscetibilidade geral aos antimicrobianos. Os patógenos multirresistentes vêm se tornando cada vez mais freqüentes nas infecções de ferida cirúrgica vascular, demonstrando variações regionais e locais quanto à suscetibilidade aos antimicrobianos profiláticos utilizados na rotina cirúrgica. Os dados e a literatura disponível até o momento demonstram que não existe evidência suficiente para uma mudança na rotina profilática perioperatória. Entretanto, devemos levar em consideração os padrões regionais e institucionais de prevalência de patógenos resistentes e padrões de suscetibilidade aos antimicrobianos para estabelecer guias e orientações específicas para a utilização de antimicrobianos profiláticos alternativos.

Palavras-chave: Antibioticoprofilaxia, infecção da ferida operatória, procedimentos cirúrgicos vasculares, cirurgia.

\section{Histórico}

As infecções cirúrgicas pós-operatórias, até a metade do século XIX, foram o grande empecilho para o progresso e desenvolvimento da cirurgia. A partir do descobrimento dos métodos anti-sépticos, em 1867, por Joseph Lister, a cirurgia experimentou sua grande evolução. Associado a outros vultos históricos e a novas descobertas, as taxas de infecção caíram de $90 \%$ para $10 \%$ até o final do século XIX ${ }^{1-3}$.

A infecção pós-operatória do sítio cirúrgico é considerada a principal causa evitável de morbimortalidade

\begin{abstract}
In peripheral vascular surgery, cephalosporins are nowadays regarded as the first choice for operative antibiotic prophylaxis. We have recently observed changes in colonizing patterns, pathogen prevalence and antibiotic susceptibility to antimicrobials. Multiresistant pathogens are becoming more frequent in vascular surgical wound infections, showing regional and local variations as to prophylactic antibiotic susceptibility. Data from the available literature so far have shown no strong evidence for a change in routine surgical antibiotic prophylaxis. We must consider regional and institutional prevalence of pathogen resistance and patterns of antibiotic susceptibility to establish specific guidelines for the use of alternative antibiotics.
\end{abstract}

Keywords: Antibiotic prophylaxis, surgical wound infection, vascular surgical procedures, surgery.

nos pacientes operados ${ }^{4}$, representando $25 \%$ de todas as infecções adquiridas no hospital ${ }^{5}$. Apesar dos avanços nos métodos anti-sépticos (material estéril, soluções anti-sépticas, lavagem das mãos), na profilaxia antimicrobiana pré-operatória e nos cuidados perioperatórios, a infecção cirúrgica pós-operatória continua sendo motivo de grande preocupação, responsável por elevada morbimortalidade e custos significativos. O custo estimado por paciente com infecção é de US\$ 2.100, gerando uma despesa anual de US\$ 4,5 bilhões ${ }^{5}$ nos EUA. A introdução dos antimicrobianos na profilaxia pré-operatória trouxe a esperança de diminuição das

1. Cirurgião vascular, Hospital Moinhos de Vento, Porto Alegre, RS. Pós-graduando, Programa de Patologia, Fundação Faculdade Federal de Ciências Médicas de Porto Alegre (FFFCMPA), Porto Alegre, RS.

2. Cirurgião vascular, Irmandade Santa Casa de Misericórdia de Porto Alegre (ISCMPA), Porto Alegre, RS. Pós-graduando, Programa de Hepatologia, FFFCMPA, Porto Alegre, RS.

3. Cirurgião plástico, ISCMPA, Porto Alegre, RS. Pós-graduando, Programa de Patologia, FFFCMPA, Porto Alegre, RS.

4. Professor adjunto, Microbiologia, FFFCMPA, Porto Alegre, RS. Professor, Programa de Pós-Graduação em Patologia, FFFCMPA, Porto Alegre, RS.

Artigo submetido em 14.07.07, aceito em 25.10.07. 
taxas de infecção nos pacientes cirúrgicos, principalmente as infecções graves ${ }^{2,3}$. Porém, atualmente observamos um aumento nos casos de infecções hospitalares graves e um crescente aumento na incidência de patógenos resistentes aos antimicrobianos utilizados.

\section{Fisiopatologia}

As infecções cirúrgicas pós-operatórias ocorrem sempre quando a combinação do número e virulência do microrganismo na ferida operatória é suficientemente grande para vencer os mecanismos de defesa locais do hospedeiro e estabelecer uma invasão dos tecidos ${ }^{1,2}$. Um estudo da década de 1960 identificou que praticamente todas as feridas cirúrgicas possuem, ao menos, um pequeno número de bactérias, mas poucas desenvolvem infecção ${ }^{6}$.

As taxas de infecção de ferida operatória publicadas na literatura são, respectivamente: 1,5 a $2,9 \%$ para as feridas limpas; 2,8 a 7,7\% para as feridas limpacontaminadas; 6,4 a 15,2\% feridas contaminadas; e de 7,1 a $40 \%$ para as feridas consideradas sujas ${ }^{7,8}$.

Os principais fatores envolvidos no desenvolvimento da infecção da ferida operatória são os bacterianos, os da ferida e os do próprio paciente. A deposição e o crescimento bacteriano são pré-requisitos para a infecção, assim como o tipo de patógeno e as toxinas produzidas pelo mesmo. Vários patógenos possuem componentes específicos que aumentam a sua virulência: as cápsulas de Klebsiella spp e do Streptococcus pneumoniae, as endotoxinas das bactérias gram-negativas, as exotoxinas dos estreptococos, o biofilme dos Staphylococcus aureus e dos Staphylococcus epidermidis. Vários estudos de feridas cirúrgicas demonstraram que, em pacientes sadios, é necessária uma contaminação com um número maior do que $10^{5}$ bactérias para a infecção ocorrer com certa freqüência. Os fatores locais incluem o material cirúrgico utilizado, técnica cirúrgica, implante de próteses, hematomas, espaço morto e cuidado com a ferida. Os fatores associados ao paciente são todas as alterações sistêmicas que podem influenciar na ferida operatória. Entre eles, citamos a idade, fluxo sanguíneo reduzido para a ferida, hipotermia, uremia, corticoesteróides, neoplasias e trauma $a^{1-3,8-11}$.

\section{Classificação das feridas cirúrgicas}

Classificação das feridas cirúrgicas de acordo com o risco de infecção:

- Ferida limpa: tem reduzido potencial de infecção; não ocorre abertura de vísceras ocas ou infração da técnica asséptica; risco de infecção de 1,5 a 2,9\% (exemplo: cirurgia vascular arterial).

- Ferida limpa-contaminada: abertura de víscera oca, com mínimo extravasamento de conteúdo ou pequenas infrações técnicas; risco de infecção de 2,8 a 7,7\% (exemplo: colecistectomia).

- Ferida contaminada: abertura de víscera oca com grosseiro extravasamento de conteúdo, inflamação aguda sem pus, infrações grosseiras na técnica asséptica e lesões traumáticas com menos de 6 horas; risco de infecção de 6,4 a 15,2\% (exemplo: colectomia).

- Ferida suja/infectada: presença de pus, víscera oca perfurada e lesões traumáticas com mais de 6 horas de evolução; risco de infecção de 7,1 a 40\% (exemplo: drenagem de abscesso) $)^{7,8}$.

\section{Métodos profiláticos}

Os quatro princípios básicos da profilaxia de infecções do sítio cirúrgico são: preparação pré-operatória do paciente, técnica cirúrgica, profilaxia antimicrobiana peri-operatória e cuidado pós-operatório com a ferida cirúrgica ${ }^{5}$. A profilaxia antimicrobiana cirúrgica é atualmente aceita como rotina na prática cirúrgica em cirurgias limpa-contaminadas, bem como em algumas cirurgias limpas. Nas feridas contaminadas e nas sujas, o antimicrobiano é sempre terapêutico e não profiláti$\mathrm{co}^{4}$. A profilaxia antimicrobiana cirúrgica deve obedecer aos princípios e indicações estabelecidos para obter sucesso, do contrário, o desenvolvimento de patógenos multirresistentes e não suscetíveis aos antimicrobianos usuais será o caminho natural. A indicação de antimicrobianos profiláticos em cirurgias simples e limpas existe apenas em casos especiais, como nas cirurgias com utilização de próteses e material sintético. Devido ao baixo risco de infecção, em torno de $1 \%$, o potencial para reduzir esse baixo índice não justifica os gastos e os efeitos colaterais da administração dos mesmos ${ }^{1,12}$. 
Tabela 1 - Tipo de cirurgia e patógenos mais freqüentes*

\begin{tabular}{ll}
\hline Tipo de cirurgia & Patógenos mais freqüentes \\
\hline Limpa & Estafilococos \\
Cardiovascular com prótese & Staphylococcus aureus, Staphylococcus coagulase-negativos \\
Biliopancreática & Bacilos gram-negativos, enterococos, anaeróbicos \\
Colorretal & Bacilos gram-negativos, Bacteroides fragilis, Escherichia coli, espécie \\
Ginecológica/obstétrica & Bacteroides, enterococos \\
Cabeça e pescoço & Escherichia coli, espécie Bacteroides, enterococos \\
\hline
\end{tabular}

* Adaptado de Lalla ${ }^{4}$.

A profilaxia antimicrobiana é mais eficaz quando iniciada no período pré-operatório e mantida no intraoperatório, com o intuito de manter níveis sanguíneos terapêuticos durante todo o procedimento. Na maioria dos procedimentos, o antimicrobiano deve ser administrado via intravenosa imediatamente antes da cirurgia, na indução anestésica. É desnecessário e prejudicial administrá-lo quando passada mais de 1 hora do início da cirurgia, bem como mantê-lo após o término da mesma. A dose única é a profilaxia padrão, dependendo, porém, do antimicrobiano administrado. No caso de cirurgias longas, a dose do antimicrobiano profilático deve ser repetida em intervalos de uma a duas meias-vidas do agente escolhido. A administração por mais de 12 horas quase nunca está indicada. Agentes profiláticos administrados algumas horas após a contaminação são muito menos efetivos, e iniciados após o término da cirurgia são totalmente desprovidos de valor $^{1-3}$.

\section{Profilaxia antimicrobiana: estado atual}

Estudos recentes têm sugerido que a incidência real de infecções pós-operatórias depois cirurgias limpas sem utilização de próteses é maior do que a relatada. Estima-se que mais de $50 \%$ das complicações ocorram após a alta do paciente, sendo subdiagnosticadas ${ }^{13-15}$. Esses casos não afetam as instituições hospitalares, mas afetam a comunidade e o sistema de saúde. Outros estudos demonstraram que pacientes submetidos a procedimentos limpos, com a utilização de antimicrobiano profilático, apresentaram menores taxas de infecção pós-operatória ${ }^{16-19}$.
O antimicrobiano utilizado como profilaxia nas infecções cirúrgicas é variado, sendo importante observar os patógenos prováveis causadores da infecção pósoperatória e determinar se haverá penetração de partes do organismo portadoras de bactérias anaeróbicas, especialmente intestinais (espécies Bacteróides). A droga de escolha em cirurgias onde não há previsão de contato com locais contaminados por bactérias anaeróbicas é a cefazolina (cefalosporina de primeira geração), que visa a cobertura principalmente aos estafilococos, principais agentes causadores de infecções em cirurgias limpas não-cavitárias ${ }^{20,21}$. Alguns autores relatam que algumas cefalosporinas de segunda geração (exemplo: cefuroxime) poderiam ser mais efetivas no tratamento dos estafilococos sensíveis à meticilina, tanto in vitro ${ }^{22}$ como na prática clínica ${ }^{23}$, porém apresentam custo significativamente mais elevado ${ }^{5}$. Nos casos onde se prevê contato com a flora intestinal, deve ser associado um antimicrobiano com atividade contra gram-negativos e anaeróbicos (aztreonan e aminoglicosídeos). Cirurgias ginecológicas e obstétricas, biliares e gastroduodenais, que possuem flora específica (Tabela 1), se beneficiam de antimicrobianos alternativos à cefazolina, como por exemplo a cefoxitina, piperacilina, ampicilina/sulbactan e amoxacilina/clavulanato ${ }^{20}$. Nos casos de alergia aos beta-lactâmicos, pode-se utilizar a eritromicina, clindamicina ou a vancomicina, devendo esta ser reservada, sempre que possível, para o tratamento de Staphylococcus aureus resistentes à meticilina ${ }^{1}$.

Atualmente, 1/3 de todas as prescrições de antimicrobianos para pacientes ambulatoriais são desnecessárias. Estudo realizado na Turquia demonstrou que em 
$23 \%$ dos pacientes o antimicrobiano estava sendo utilizado de forma incorreta ${ }^{24}$. Uma revisão, incluindo 44 hospitais de Nova Iorque, EUA, demonstrou que foram utilizados 44 diferentes antimicrobianos na profilaxia pré-operatória e, apesar da profilaxia ter sido utilizada em $81-94 \%$ dos pacientes, $27-54 \%$ foram administradas no período incorreto ${ }^{5}$.

\section{Resistência aos antimicrobianos}

Devido ao surgimento de patógenos multirresistentes, à diminuição generalizada da suscetibilidade aos antimicrobianos e à mudança do perfil dos patógenos colonizantes comunitários e hospitalares, a questão mais importante atualmente é se os antimicrobianos clássicos, utilizados com profilaxia cirúrgica, ainda seguem sendo o padrão-ouro na prevenção das infecções cirúrgicas pós-operatórias, principalmente quando comparadas à vancomicina e à teicoplanina. O Center for Disease Control and Prevention (CDC) estadunidense não indica o uso rotineiro de vancomicina como profilaxia antimicrobiana para nenhum tipo de procedimento cirúrgico ${ }^{21}$. Certamente existem exceções, principalmente nos casos onde o hospital ou a instituição apresenta taxas maiores de $20 \%$ de infecções pósoperatórias causadas por Staphylococcus aureus resistente à meticilina $(\mathrm{MRSA})^{25}$. Nestes casos, a profilaxia antimicrobiana cirúrgica deverá ser conduzida com a utilização de vancomicina ou teicoplanina.

Atualmente, com a execução de procedimentos cada vez mais complexos e prolongados, transplantes em pacientes imunodeprimidos, cirurgias em pacientes com múltiplas comorbidades e cirurgias com implante de materiais protéticos, a profilaxia antimicrobiana préoperatória visando a proteção contra os patógenos resistentes vem se tornando um desafio.

\section{Principais antimicrobianos utilizados na profilaxia}

\section{Cefalosporinas de primeira geração}

A cefazolina é uma cefalosporina de primeira geração de meia-vida curta e administração parenteral que faz parte da família dos antibióticos beta-lactâmicos. Atua primariamente pela inibição de um passo na síntese da parede celular bacteriana (transpeptidação), que resulta na lise celular bacteriana espontânea. Os mecanismos de resistência mais importantes são a produção de beta-lactamases, que provocam hidrólise do anel betalactâmico, a modificação genética nas proteínas que se ligam à penicilina (PBPs), os receptores bacterianos para as drogas beta-lactâmicas. Os efeitos adversos mais comuns são hipersensibilidade, desconforto gastrintestinal e desenvolvimento de resistência bacteriana ${ }^{26,27}$.

$\mathrm{Na}$ década de 60 foi introduzido o conceito de profilaxia perioperatória, revolucionando os critérios de tratamento antimicrobiano existentes até o momento. Esse método permitiu reduzir os fenômenos de sepse perioperatória e diminuir o alto custo do tratamento hospitalar por complicações infecciosas. Foram demonstradas nessa época vantagens em relação ao custo-benefício do uso da cefazolina. A avaliação clínica prospectiva mostrou que a profilaxia antimicrobiana com $1 \mathrm{~g}$ de cefazolina intravenosa em dose única no pré-operatório foi eficaz na redução dos níveis de infecção em cirurgias que apresentavam complicações infecciosas em taxa superior a $7 \%{ }^{28-30}$. Atualmente, a prática de utilizar antimicrobianos de maneira profilática está bem difundida, estando presente em mais de $90 \%$ dos procedimentos cirúrgicos $^{31}$.

A profilaxia antimicrobiana tem sua indicação em procedimentos limpos e limpo-contaminados, de preferência com um só tipo de antimicrobiano, iniciando-se a aplicação da droga de escolha no período pré-operatório $^{32}$.

Foi realizado um estudo multicêntrico para detectar o tempo necessário para obter uma profilaxia perioperatória eficiente com o uso de cefazolina. Comparou-se a eficácia de uma dose única de profilaxia com outros esquemas de doses múltiplas. Um total de 527 pacientes foi tratado para neoplasias de pele e em áreas propensas à contaminação. Os grupos foram divididos em: A) sem profilaxia; B) cefazolina $1 \mathrm{~g}$, de 12/12 horas, começando 48 horas previamente à cirurgia e continuado por 48 horas depois da cirurgia; C) cefazolina 1, g de 12/12 horas, intramuscular e começando 2 horas antes da cirurgia e continuado por 24 horas após a cirurgia; e D) cefazolina intramuscular, $1 \mathrm{~g}$ em dose única, 2 horas antes da cirurgia. A taxa de infecções no pós-operatório foi, no grupo A, $12 \%$; grupo B, 4,6\%; grupo C, 0,77\%; e 
grupo D, 2,96\%. O estudo confirmou a utilidade do antimicrobiano profilático na prevenção das infecções pósoperatórias e demonstrou que os regimes curtos são superiores aos mais prolongados. Em particular, a dose única de antimicrobiano, que reduziu significativamente a taxa de infecção, sendo mais barata e melhor tolerada pelos pacientes ${ }^{33}$. Os achados anteriores foram confirmados por outros estudos, envolvendo múltiplos tipos de procedimentos, mostrando que uma dose única de cefazolina no pré-operatório diminui os custos do tratamento e mantém a eficácia na prevenção de eventos infecciosos ${ }^{34}$.

No intuito de verificar o efeito da profilaxia com cefazolina na taxa de infecção de ferida operatória em feridas limpas de acordo com o estado clínico pré-operatório, foi realizado um ensaio clínico randomizado duplo-cego com um total de 303 pacientes. Foram agrupados os casos de acordo com a classificação ASA (American Society of Anesthesiologists). Os pacientes ASA 2 e ASA 3 se beneficiaram da profilaxia antimicrobiana, mostrando que os pacientes que não receberam o antimicrobiano tiveram 4,3 e 4,8 vezes, respectivamente, maior risco de infecção do que no grupo controle (risco relativo 0,91 ; intervalo de confiança de $0,83-0,99 ; \mathrm{p}=0,02)^{35}$.

Em procedimentos limitados às partes moles e superficiais, foi desenhado estudo de caso-controle para identificar os fatores de risco para infecção de ferida operatória (FO). Os controles utilizados foram todos os pacientes que realizaram cirurgias estéticas e não desenvolveram infecção de FO. Doze pacientes do grupo controle e 4 pacientes que desenvolveram infecção de FO (por Staphylococcus aureus) foram incluídos no estudo. Os fatores de risco associados à infecção de $\mathrm{FO}$ foram: a média de duração do procedimento $(5 \mathrm{~h} v s .2 \mathrm{~h} ; \mathrm{p}=$ $0,02)$; anestesia geral $(\mathrm{p}=0,004)$; e colocação de drenos $(p=0,004)$. No mesmo estudo, após a reintrodução da profilaxia antimicrobiana com cefazolina para procedimentos de duração estimada maior de 3 horas, a taxa de infecção de $\mathrm{FO}$ foi nula ${ }^{36}$. Em contrapartida, existem evidências de que, em determinados tipos de intervenção, a profilaxia antimicrobiana parece não trazer benefícios.
Um estudo realizado para determinar a utilidade de antimicrobianos profiláticos, em operações de fístulas artério-venosas para diálise, demonstrou que a única infecção local pós-operatória ocorreu em um paciente que tinha recebido a profilaxia. Os autores concluíram que a profilaxia antimicrobiana é desnecessária em operações de fístula artério-venosa para diálise. Com ou sem antimicrobiano, a taxa de infecção é quase zero ${ }^{37}$.

Há uma preocupação com o desenvolvimento de resistência bacteriana à cefazolina. Existe uma prevalência aproximada de 30\% de portadores de Staphylococcus aureus, sendo mais de 96\% desses resistentes à meticilina ${ }^{38}$. O programa SENTRY, em 1997, demonstrou uma incidência de 16,7\% de Staphylococcus aureus resistente à meticilina ${ }^{39}$.

Estudos mais atuais são necessários para verificar a taxa de resistência à cefazolina em infecções de FO limpas e limpa-contaminadas, inclusive as limitadas às partes moles e pele, tanto com programas mundiais quanto locais, para que o estado atual da eficácia da cefazolina como agente profilático seja estabelecido e novas opções de antimicrobianos sejam padronizados ${ }^{40-43}$.

\section{Antimicrobianos alternativos utilizados na profilaxia}

A utilização disseminada de alguns antimicrobianos, como as cefalosporinas de primeira e segunda gerações, em regime profilático e terapêutico resultou em um aumento dramático na prevalência da resistência bacteriana ${ }^{44}$. Nesse contexto, ouve um aumento da prevalência de organismos multirresistentes, como o Staphylococcus aureus MRSA $^{45}$. Em ambientes ou instituições com alta prevalência de bactérias resistentes à meticilina, antimicrobianos alternativos, ou seja, de utilização não rotineira ou consagrada, como os glicopeptídeos, são a primeira escolha como agentes profiláticos ${ }^{46,47}$.

\section{Vancomicina}

A vancomicina foi introduzida na prática clínica na década de 1950, e durante as três décadas seguintes a resistência bacteriana à mesma foi raramente relata$\mathrm{da}^{44}$. No entanto, na década de 80 , observamos o surgimento de estafilococos coagulase-negativos resistentes à vancomicina, especialmente o Staphylococcus epidermidis, Staphylococcus hominis, Staphylococcus warneri, 
Staphylococcus haemolyticus e Staphylococcus xylosus $^{48,49}$. O CDC estadunidense recomenda que a vancomicina deve ser utilizada apenas como agente profilático nos casos em que existe cepa de MRSA ou estafilococo coagulase-negativo resistente à meticilina em infecção de ferida cirúrgica ou quando existe elevada prevalência de MRSA isolados no local ou instituição ${ }^{21}$. Infelizmente, as orientações do CDC não atribuem nenhum valor à prevalência de resistência à meticilina que justificaria a profilaxia com glicopeptídeos. Zanetti et al. ${ }^{50}$ demonstraram que seria necessária uma performance superior da cefazolina em relação aos organismos suscetíveis, a menos que a prevalência de resistência à meticilina fosse menor do que $3 \%$. Comparando a profilaxia antimicrobiana com vancomicina e cefazolina na cirurgia de fratura de colo do fêmur, Merrer et al. ${ }^{51}$ obtiveram uma incidência similar de infecções do sítio cirúrgico em pacientes que receberam cefazolina (4\%) e vancomicina ( $2 \%)$. Além disso, os mesmos autores observaram que o impacto de ambos antimicrobianos no surgimento de cepas de enterococos e estafilococos resistentes aos glicopeptídeos foi semelhante. Acredita-se que a utilização de vancomicina promova o desenvolvimento e a transmissão dessa resistência ${ }^{52}$. Além disso, a vancomicina também é mais dispendiosa e de difícil administração se comparada à cefazolina, sendo a primeira escolha apenas na prevenção de infecções por MRSA e estafilococos coagulase-negativos resistentes à meticili$\mathrm{na}^{52}$. Entretanto, Zanetti et al. ${ }^{50}$ demonstraram que a profilaxia de rotina com vancomicina foi mais efetiva do que com a cefazolina em pacientes submetidos à cirurgia de revascularização do miocárdio, tendo prevenido um maior número de infecções de ferida cirúrgica ou óbitos por estafilococos e enterococos resistentes à meticilina. Além disso, a utilização rotineira de vancomicina foi menos dispendiosa do que a cefazolina, sendo que nem os maiores custos de compra e administração e nem a ausência de proteção contra os gram-negativos diminuíram o saldo positivo final. Os autores comentam que a utilização da vancomicina nos EUA poderia prevenir 110 óbitos e 3.184 infecções de ferida cirúrgica quando comparada à utilização da cefazolina. Por outro lado, é difícil recomendar o uso universal da vancomicina devido aos dados insuficientes quanto às possíveis conseqüências do uso rotineiro de vancomicina e seu impacto no desenvolvimento da resistência bacteriana.
Algumas vezes, a administração sistêmica dos antimicrobianos é insuficiente para prevenir a infecção de prótese, pois a concentração do antimicrobiano no tecido em volta da prótese é muito baixa. Hirose et al. ${ }^{53}$ desenvolveram, recentemente, um sistema de liberação sustentada do antimicrobiano através da utilização de caprolactona (material biodegradável) que mantém uma concentração tecidual efetiva do antimicrobiano em volta do enxerto protético. Os mesmos autores demonstraram que a liberação sustentada de vancomicina reduziu a taxa de infecção em próteses em modelo animal.

\section{Teicoplanina}

Os microrganismos mais freqüentes causadores de infecção no sítio cirúrgico de cirurgias ortopédicas e vasculares são os cocos gram-positivos, com predomínio do Staphylococcus spp, sendo responsáveis por $70-90 \%$ dos patógenos isolados. A maior razão para esse predomínio é a habilidade desses patógenos com relação à aderência e multiplicação em polímeros através da produção do biofilme ${ }^{54}$. Os glicopeptídeos têm sido considerados uma alternativa razoável, especialmente em uma era de elevada prevalência de estafilococos resistentes à meticilina $^{55}$. Vários estudos têm comparado a efetividade e a toxicidade da teicoplanina e das cefalosporinas como profilaxia antimicrobiana pré-operatória, mas os resultados foram inconclusivos. Kardakas et al. ${ }^{55}$ conduziram uma metanálise, comparando a eficácia e segurança da teicoplanina e das cefalosporinas de primeira (cefazolina) e segunda geração na profilaxia antibacteriana pré-operatória em cirurgia ortopédica e vascular. Foram identificados dois estudos envolvendo procedimentos vasculares e quatro envolvendo procedimentos ortopédicos entre janeiro de 1950 e novembro de 2004, totalizando 510 pacientes submetidos à cirurgia vascular e 2.376 submetidos à cirurgia ortopédica. Os autores não observaram nenhuma diferença entre a teicoplanina e as cefalosporinas quanto ao desenvolvimento de infecção da ferida cirúrgica ou em outros sítios. Adicionalmente, não foi observada nenhuma diferença quanto aos efeitos adversos ou mortalidade.

A teicoplanina possui uma meia-vida de 45-70 horas, podendo ser administrada em dose única. Em contraste, as cefalosporinas de primeira e segunda geração necessitam de regimes com múltiplas doses ${ }^{56}$. Além 
disso, o espectro antimicrobiano da teicoplanina cobre o estafilococo resistente à meticilina, que é parte da flora normal de $25 \%$ dos pacientes submetidos à cirurgia com colocação de prótese articular total ${ }^{57}$. Essas propriedades dão suporte à seleção dos glicopeptídeos como agentes profiláticos pré-operatórios em cirurgias ortopédicas e vasculares, envolvendo a utilização de material protético. Portanto, não é de se surpreender que tanto a vancomicina como a teicoplanina estejam sendo utilizadas em larga escala, em vários países, com esse propósito. Entretanto, os achados descritos acima sugerem que não há superioridade de um antimicrobiano sobre o outro com relação à prevenção de infecção, desenvolvimento de efeitos adversos e mortalidade total.

Com relação ao glicopeptídeo a ser utilizado, a escolha inicial para a profilaxia cirúrgica recai sobre a teicoplanina devido à sua excelente penetração tecidual, demonstrada pela ótima distribuição, baixa toxicidade e meia-vida prolongada. Vários ensaios clínicos, utilizando a teicoplanina como agente profilático préoperatório, em cirurgias limpas ortopédicas, cardíacas, vasculares e dentárias, demonstraram a sua eficácia ${ }^{58}$.

\section{Antimicrobianos que se ligam aos enxertos protéticos}

Como medida profilática adicional, em casos onde será utilizada especificamente uma prótese vascular, tem sido proposta a utilização de antimicrobianos que se liga ao enxerto protético em altas concentrações ${ }^{59-61}$. No caso da cirurgia vascular, vários antimicrobianos têm sido propostos como profilaxia adjunta ${ }^{62,63}$. Estudos clínicos vêm utilizando próteses de Dacron impregnadas com rifampicina com o intuito de prevenir a colonização e infecção das mesmas ${ }^{62}$. Outros tipos de enxerto impregnados com antimicrobianos têm sido utilizados apenas em estudos experimentais ${ }^{45}$. Vicaretti et al. ${ }^{64}$ estabeleceram um modelo animal de infecção por Staphylococcus epidermidis em prótese vascular, sugerindo que um aumento na concentração de rifampicina ligada à prótese (Dacron) reduziria significativamente a incidência de infecção de prótese vascular ocasionada por estafilococos resistentes. Por outro lado, o desenvolvimento de resistência à rifampicina, com a utilização em larga escala dessas próteses impregnadas, resultaria na necessidade do desenvolvimento de novos e complexos métodos profiláticos ${ }^{65}$.
A mupirocina, produzida pela Pseudomonas fluorescens, é um antimicrobiano tópico utilizado no tratamento de infecções superficiais de pele ocasionadas pelos estafilococos (Staphylococcus aureus e Streptococcus pyogenes) e para a erradicação do Staphylococcus aureus nasal (colonizante) ${ }^{66}$. A mupirocina foi introduzida na prática clínica em 1985, no Reino Unido, mas o desenvolvimento de resistência foi descrito logo após o início da sua utilização clínica ${ }^{67}$. Giacometti et al. ${ }^{44}$ investigaram, em modelos animais, a eficácia da mupirocina na prevenção da infecção de prótese vascular por cepas de Staphylococcus epidermidis com diferentes padrões de resistência (suscetível à meticilina, resistente à meticilina e resistência intermediária à vancomicina). Os autores demonstraram que o uso da prótese de Dacron impregnada com mupirocina pode resultar em uma inibição significativa do crescimento estafilocócico, mesmo quando esses forem multirresistentes. Outros autores demonstraram a superioridade da mupirocina sobre a rifampicina na prevenção de infecções por MRSA ${ }^{65}$.

Mais recentemente, estudos envolvendo a utilização de estreptograminas (quinupristin/dalfopristin) têm sido conduzidos em modelos animais, buscando avaliar a sua capacidade de prevenção de infecções por Staphylococcus epidermidis resistente à meticilina e com resistência intermediária aos glicopeptídeos. No estudo de Giacometti et al. ${ }^{68}$ foi observada uma significativa redução no crescimento bacteriano em próteses impregnadas com a nova droga, in vivo.

Outro estudo recente avaliou a eficácia de próteses impregnadas com uma associação de vancomicina, teicoplanina e ácido fusídico na prevenção de infecções de prótese, modelo animal. Os autores demonstraram que a associação do ácido fusídico aos glicopeptídeos resultou em uma inibição significativamente maior do crescimento bacteriano de MRSA, mesmo quando cepas multirresistentes eram inoculadas diretamente na prótese $^{45}$.

O desenvolvimento de próteses resistentes à infecção possui um apelo comercial forte, mas até o momento nenhum estudo confirmou in vivo os resultados obtidos in vitro. Entretanto, a utilização de próteses impregnadas com antimicrobianos poderá ser uma medida 
adjunta importante no futuro para a profilaxia antimicrobiana em cirurgias que utilizam material sintético.

\section{Conclusão}

Apesar das cefalosporinas estarem consagradas como agente profilático antimicrobiano pré-operatório, devemos atentar para a recente mudança dos padrões de colonização e suscetibilidade aos antimicrobianos. Atualmente, os patógenos multirresistentes vêm se tornando cada vez mais freqüentes em infecções de ferida cirúrgica, demonstrando variações regionais e até locais quanto à suscetibilidade aos agentes antimicrobianos profiláticos utilizados de rotina. Concluímos que não existe evidência suficiente que justifique uma mudança na profilaxia antimicrobiana cirúrgica clássica. No entanto, os padrões regionais e institucionais de prevalência de patógenos resistentes e suscetibilidade aos antimicrobianos devem nortear a tomada de decisão para a utilização de antimicrobianos alternativos na profilaxia pré-operatória de forma individualizada.

\section{Referências}

1. Dellinger EP. Infecções cirúrgicas e escolha dos antimicrobianos. In: Townsend CM, editor. Sabiston: tratado de cirurgia. $6^{\mathrm{a}}$ ed. Rio de Janeiro: Guanabara Koogan; 2003. p. 182-200.

2. Fry DE, editor. Surgical infections. Boston: Little Brown; 1995.

3. Howard RJ, Simmons RL, editors. Surgical infectious diseases. Norwalk: Appleton-Lange; 1994.

4. de Lalla F. Surgical prophylaxis in practice. J Hosp Infect. 2002;50(SupplA):S9-12.

5. Akalin HE. Surgical prophylaxis: the evolution of guidelines in an era of cost containment. J Hosp Infect. 2002;50(SupplA):S3-7.

6. Burke JF. Identification of the source of staphylococci contaminating the surgical wound during operation. Ann Surg. 1963;158:898-904.

7. Dellinger EP, Ehrenkranz NJ. Surgical infections. In: Bennett JV, Brachman PS, editors. Hospital infections. 4th ed. Philadelphia: Lippincott-Raven; 1998. p. 571-85.

8. Cruse PJ, Foord R. The epidemiology of wound infection. A prospective study of 62,939 wounds. Surg Clin North Am. 1980;60:27-40.

9. Linton RR. The prophylactic use of the antibiotics in clean surgery. Surg Gynecol Obstet. 1961;112:218-20.

10. Greif R, Akça O, Horn EP, Kurz A, Sessler DI. Supplemental perioperative oxygen to reduce the incidence of surgicalwound infection. Outcomes Research Group. N Engl J Med. 2000;342:161-7.
11. Kurz A, Sessler DI, Lenhardt R. Perioperative normothermia to reduce the incidence of surgical-wound infection and shorten hospitalization. Study of Wound Infection and Temperature Group. N Engl J Med. 1996;334:1209-15.

12. Page CP, Bohnen JM, Fletcher JR, McManus AT, Solomkin JS, Wittmann DH. Antimicrobial prophylaxis for surgical wounds: Guidelines for clinical care. Arch Surg. 1993;128:7988.

13. Manian FA, Meyer L. Comprehensive surveillance of surgical wound infections in outpatient and inpatient surgery. Infect Control Hosp Epidemiol. 1990;11:515-20.

14. Olson MM, Lee JT Jr. Continuous, 10-year wound infection surveillance. Results, advantages, and unanswered questions. Arch Surg. 1990;125:794-803.

15. Bailey IS, Karran SE, Toyn K, Brough P, Ranaboldo C, Karran SJ. Community surveillance of complications after hernia repair surgery. BMJ. 1992;304:469-71.

16. Platt R, Zaleznik DF, Hopkins CC, et al. Perioperative antibiotic prophylaxis for herniorrhaphy and breast surgery. $\mathrm{N}$ Engl J Med. 1990;322:153-60.

17. Platt R, Zucker JR, Zaleznik DF, et al. Prophylaxis against wound infection following herniorrhaphy or breast surgery. J Infect Dis. 1992;166:556-60.

18. Lewis RT, Weigand FM, Mamazza J, Lloyd-Smith W, Tataryn D. Should antibiotic prophylaxis be used routinely in clean surgical procedures: a tentative yes. Surgery. 1995;118:742-6.

19. Leaper D. Use of antibiotic prophylaxis in clean non-implant wounds. J Antimicrob Chemother. 1998;41:501-4.

20. de Lalla F. Antimicrobial chemotherapy in the control of surgical infectious complications. J Chemother. 1999;11:440-5.

21. Mangram AJ, Horan TC, Pearson ML, Silver LC, Jarvis WR. Guideline for prevention of surgical site infection. Am J Infect Control. 1999;27:97-132.

22. Kernodle DS, Classen DC, Burke JP, Kaiser AB. Failure of cephalosporins to prevent Staphylococcus aureus surgical wound infections. JAMA. 1990;263:961-9.

23. Kreter B, Woods M. Antibiotic prophylaxis for cardiothoracic operations. Meta-analysis of thirty years of clinical trials. J Thorac Cardiovasc Surg. 1992;104:590-9.

24. Pehlivan M, Hayran M, Akalin HE. Antibacterial use at Iiacettepe University Hospital: Differences between specialities on appropriate use. Symposium on Nosocomial Infections, Turkey, 1994.

25. Fraise AP. Guidelines for the control of methicillin-resistant Staphylococcus aureus. J Antimicrob Chemother. 1998;42:287-9.

26. Katzung BG, Ehrlich KS. Drogas utilizadas em infecções bacterianas, fúngicas e virais. In: Katzung B, editor. Farmacologia clínica. $1^{\mathrm{a}}$ ed. Porto Alegre: Artmed; 1991. p. 132-80.

27. Tice AD. Pharmacoeconomic considerations in the ambulatory use of parenteral cephalosporins. Drugs. 2000;59:29-35.

28. Barrionuevo CE, Koning MB, Capasso R, Barrionuevo R. Eficácia do uso da cefazolina per-operatória em dose única na profilaxia da infecçäo pós-operatória em cirurgias näo infectadas. . Rev Bras Med Otorrinolaringol. 1995;2:432,434-5. 
29. Moya M, Antonio D, Collazo Herrera M, Pisonero Socías J, Pardo Gómez G. Evaluación económica del uso de Cefazolina versus Ceftriazona en la profilaxis perioperatoria. Rev cuba Farm. 2001;35:187-191.

30. Valle Panchana G, Abarca Aguilar F, Rivera Escalante V, Rivera Drouet R. Cefazolina y su uso como profilaction en cirurgia. Rev Univ Guayaquil. 1987;2:59-68.

31. Baca Tinoco C. Uso de antibiótico para profilaxis quirúrgica en el servicio de cirugía general del HEODRA, en el período enero a diciembre 2003. León. 2004; s.n:45.

32. Saab A, Suckerman-Voldman E, Rodríguez AR, et al. Profilaxia con antibióticos en cirugía: experiencia en el Servicio de Emergencia de un Hospital Universitário. Antibiot Infecc. 1995;5:13-6.

33. Bencini PL, Signorini M, Galimberti M, Cavicchini S, Caputo R. Preoperative antibiotic prophylaxis in flexural surgery of difficult contamination-prone areas of the skin: the utility of a single dose of antibiotic. J Dermatol Treat. 1994;5:17-9.

34. Fonseca SN, Kunzle SR, Junqueira MJ, Nascimento RT, de Andrade JI, Levin AS. Implementing 1-dose antibiotic prophylaxis for prevention of surgical site infection. Arch Surg. 2006;141:1109-14.

35. Iribarren O, Araujo M. Effect of antimicrobial prophylaxis on the incidence of infections in clean surgical wounds in hospitals undergoing renovation. Infection control and hospital epidemiology. Infect Control Hosp Epidemiol. 2006;27:1372-6.

36. Fatica CA, Gordon SM, Zins JE. The role of preoperative antibiotic prophylaxis in cosmetic surgery. Plast Reconstr Surg. 2002;109:2570-3.

37. Moreira RC, Timi JR, Abrão E. Estudo prospectivo da antibioticoprofilaxia em operaçöes de fístula artério-venosa para diálise. Rev Angiol Cir Vasc. 1992;1:108-11.

38. Del Canto Harboe E, Urbina CR. Estudio de portación nasal de staphylococcus aureus en estudiantes de medicina de la Universidad de Santiago de Chile. Clin Cienc. 2001;1:10-14.

39. Robledo JÁ, López J, Sierra P, Robledo C, Pfaller MA, Jones RN. El programa de vigilancia antimicrobiana SENTRY en Colombia: hallazgos iniciales en tres hospitales de Medellín. Infectio. 1999;3:100-7.

40. Terzi C, Kilic D, Unek T, Hosgorler F, Fuzun M, Ergor G. Single-dose oral ciprofloxacin compared with single-dose intravenous cefazolin for prophylaxis in inguinal hernia repair: a controlled randomized clinicalstudy. J Hosp Infect. 2005;60:340-7.

41. Thomas R, Alvino P, Cortino G R, et al. Long-acting versus short-acting cephalosporins for preoperative prophylaxis in breast surgery: a randomized double-blind trial involving 1,766. Chemotherapy. 1999;45:217-23.

42. Marroni M, Cao P, Fiorio M, et al. Prospective, randomized, double-blind trial comparing teicoplanin and cefazolin as antibiotic prophylaxis in prosthetic vascular surgery. Eur $\mathbf{J}$ Clin Microbiol Infect Dis. 1999;18:175-8.

43. Allababidi S, Shah JC. Efficacy and pharmacokinetics of sitespecific cefazolin delivery using biodegradable implants in the prevention of post-operative wound infections. Pharm Res. 1998;15:325-33.
44. Giacometti A, Cirioni O, Ghiselli R, et al. Mupirocin prophylaxis against methicillin-susceptible, methicillin-resistant, or vancomycin-intermediate Staphylococcus epidermidis vascular-graft infection. Antimicrob Agents Chemother. 2000; 44:2842-4.

45. Yasim A, Gul M, Atahan E, Ciragil P, Aral M, Ergun Y. Efficacy of vancomycin, teicoplanin and fusidic acid as prophylactic agents in prevention of vascular graft infection: an experimental study in rat. Eur J Vasc Endovasc Surg. 2006;31:274-9.

46. Earnshaw JJ. Methicillin-resistant Staphylococcus aureus: vascular surgeons should fight back. Eur J Vasc Endovasc Surg. 2002;24:283-6.

47. Murphy GJ, Pararajasingam R, Nasim A, Dennis MJ, Sayers RD. Methicillin-resistant Staphylococcus aureus infection in vascular surgical patients. Ann R Coll Surg Engl. 2001;83:158-63.

48. McManus AT, Goodwin CW, Pruitt BA Jr. Observations on the risk of resistance with the extended use of vancomycin. Arch Surg. 1998;133:1207-11.

49. Schwalbe RS, Stapleton JT, Gilligan PH. Emergence of vancomycin resistance in coagulase-negative staphylococci. $\mathrm{N}$ Engl J Med. 1987;316:927-31.

50. Zanetti G, Goldie SJ, Platt R. Clinical consequences and cost of limiting use of vancomycin for perioperative prophylaxis: example of coronary artery bypass surgery. Emerg Infect Dis. 2001;7:820-7.

51. Merrer J, Desbouchages L, Serazin V, Razafimamonjy J, Pauthier F, Leneveu M. Comparison of routine prophylaxis with vancomycin or cefazolin for femoral neck fracture surgery: microbiological and clinical outcomes. Infect Control Hosp Epidemiol. 2006;27:1366-71.

52. Martone WJ. Spread of vancomycin-resistant enterococci: why did it happen in the United States? Infect Control Hosp Epidemiol. 1998;19:539-45.

53. Hirose K, Marui A, Arai Y, et al. Sustained-release vancomycin sheet may help to prevent prosthetic graft methicillinresistant Staphylococcus aureus infection. J Vasc Surg. 2006;44:377-82.

54. de Lalla F. Antibiotic prophylaxis in orthopedic prosthetic surgery. J Chemother. 2001;1:48-53.

55. Vardakas KZ, Soteriades ES, Chrysanthopoulou1 A, Papagelopoulos PJ, Falagas ME. Perioperative anti-infective prophylaxis with teicoplanin compared to cephalosporins in orthopaedic and vascular surgery involving prosthetic material. Clin Microbiol Infect. 2005;11:775-7.

56. de Lalla F, Novelli A, Pellizzer G, et al. Regional and systemic prophylaxis with teicoplanin in monolateral and bilateral total knee replacement procedures: study of pharmacokinetics and tissue penetration. Antimicrob Agents Chemother. 1993;37:2693-8.

57. Nehrer S, Thalhammer F, Schwameis E, Breyer S, Kotz R. Teicoplanin in the prevention of infection in total hip replacement. Arch Orthop Trauma Surg. 1998;118:32-6.

58. Mini E, Nobili S, Periti P. Does surgical prophylaxis with teicoplanin constitute a therapeutic advance? J Chemother. 2000;12:40-55. 
59. Chervu A, Moore WS, Gelabert HA, Colburn MD, Chvapil M. Prevention of graft infection by use of prostheses bonded with a rifampin/collagen release system. J Vasc Surg. $1991 ; 14: 521-4$.

60. Harvey RA, Alcid DV, Greco RS. Antibiotic bonding to polytetrafluoroethylene with tridodecylmethylammonium chloride. Surgery. 1982;92:504-12.

61. Osada T, Yamamura K, Fujimoto K, et al. Prophylaxis of local vascular graft infection with levofloxacin incorporated into albumin-sealed Dacron graft. Microbiol Immunol. 1999;43:317-21.

62. Bandyk D F, Novotney ML, Back MR, Johnson BL, Schmacht DC. Expanded application of in situ replacement for prosthetic graft infection. J Vasc Surg. 2001;34:411-20.

63. Goeau-Brissonniere O, Leport C, Bacourt F, Lebrault C, Comte R, Pechere JC. Prevention of vascular graft infection by rifampin bonding to a gelatin-sealed Dacron graft. Ann Vasc Surg. 1991;5:408-12.

64. Vicaretti M, Hawthorne WJ, Ao PY, Fletcher JP. An increased concentration of rifampicin bonded to gelatin-sealed Dacron reduced the incidence of subsequent graft infections following a staphylococcal challenge. Cardiovasc Surg. 1998;6:268-73.
65. Ghiselli R, Giacometti A, Goffi L, et al. Prophylaxis against Staphylococcus aureus vascular graft infection with mupirocin-soaked, collagen-sealed Dacron. J Surg Res. 2001;99:316-20.

66. Sutherland R, Boon RJ, Griffin KE, Masters PJ, Slocombe B, White AR. Antibacterial activity of mupirocin (pseudomonic acid), a new antibiotic for topical use. Antimicrob Agents Chemother. 1985;27:495-8.

67. Cookson BD. The emergence of mupirocin resistance: a challenge to infection control and antibiotic prescribing practice. J Antimicrob Chemother. 1998;41:11-8.

68. Giacometti A, Cirioni O, Ghiselli R, et al. Efficacy of quinupristin-dalfopristin in preventing vascular graft infection due to Staphylococcus epidermidis with intermediate resistance to glycopeptides. Antimicrob Agents Chemother. 2002;46:2885-8.

Correspondência:

Eduardo Lichtenfels

Rua Honório S. Dias, 1500/305

CEP 90540-070 - Porto Alegre, RS

Tel.: (51) 3325.5379

E-mail: elichtenfels@uol.com.br 